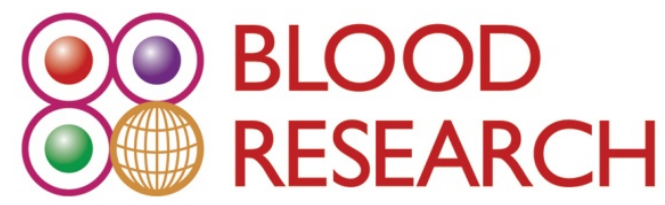

\title{
Real-world evidence of levofloxacin prophylaxis in elderly patients with newly diagnosed multiple myeloma who received bortezomib, melphalan, and prednisone regimen
}

Su-In Kim, Sung-Hoon Jung, Ho-Young Yhim, Jae-Cheol Jo, Ga-Young Song, Mihee Kim, Seo-Yeon Ahn, Jae-Sook Ahn, Deok-Hwan Yang, Hyeoung-Joon Kim, Je-Jung Lee

Citation : Kim SI, Jung SH, Yhim HY, et al. Real-world evidence of levofloxacin prophylaxis in elderly patients with newly diagnosed multiple myeloma who received bortezomib, melphalan, and prednisone regimen. Blood Res 2022. [Epub ahead of print]

Publisher's disclaimer: This manuscript has completed peer review and has been accepted for publication. We are distributing the authors' version of manuscript prior to technical and English editing, typesetting and proof reading. A final version approved by the authors' will follow in a regular issue of BLOOD RESEARCH. All legal disclaimers that apply to BLOOD RESEARCH also pertain to this production process. 


\section{Real-world evidence of levofloxacin prophylaxis in elderly patients with newly diagnosed multiple myeloma who received bortezomib, melphalan, and prednisone regimen}

Su-In Kim ${ }^{1 *}$, Sung-Hoon Jung ${ }^{1 *}$, Ho-Young Yhim², Jae-Cheol Jo ${ }^{3}$, Ga-Young Song ${ }^{1}$, Mihee Kim ${ }^{1}$, Seo-Yeon $\mathrm{Ahn}^{1}$, Jae-Sook Ahn ${ }^{1}$, Deok-Hwan Yang ${ }^{1}$, Hyeoung-Joon Kim ${ }^{1}$, Je-Jung Lee ${ }^{1}$

${ }^{1}$ Department of Hematology-Oncology, Chonnam National University Hwasun Hospital and Chonnam National University Medical School, Hwasun, ${ }^{2}$ Jeonbuk National University Hospital, Jeonbuk National university Medical School, Jeonju, ${ }^{3}$ Department of Internal Medicine, Ulsan University Hospital, University of Ulsan College of Medicine, Ulsan, Korea

*These authors contributed equally to this work.

Correspondence to: Je-Jung Lee, M.D., Ph.D.

Department of Hematology-Oncology, Chonnam National University Hwasun Hospital, 322 Seoyangro, Hwasun, Jeonnam 58128, Korea

E-mail: drjejung@,chonnam.ac.kr 


\begin{abstract}
Background: Although survival outcomes of multiple myeloma (MM) have improved with the development of new and effective agents, infection remains the major cause of morbidity and mortality. Here, we evaluated the efficacy of levofloxacin prophylaxis (in a real-world setting) during bortezomib, melphalan, and prednisone (VMP) therapy in elderly patients with newly diagnosed MM. Methods: This study retrospectively analyzed the records of patients with newly diagnosed MM treated with the VMP regimen between February 2011 and September 2020 at three institutes of the Republic of Korea.

Results: Of a total of 258 patients, 204 (79.1\%) received levofloxacin prophylaxis during VMP therapy. The median number of levofloxacin prophylaxis cycles was 4 (range, 1-9), but 10 patients did not complete the planned prophylaxis because of side effects. Sixty-six patients (25.5\%) experienced severe infections during VMP therapy, most of which (74.7\%) occurred within the first four cycles of VMP therapy regardless of levofloxacin prophylaxis status. Early severe infection was significantly associated with poor survival. In multivariate analysis, levofloxacin prophylaxis was significantly associated with a lower risk in early severe infection.
\end{abstract}

Conclusion: Our findings suggest that levofloxacin prophylaxis should be considered at least during the first four cycles of VMP therapy in elderly patients with newly diagnosed MM.

Keywords: Early infection, Prophylaxis, Levofloxacin, Multiple myeloma 


\section{INTRODUCTION}

Multiple myeloma (MM) is the second most common hematological malignancy, characterized by clonal expansion of malignant plasma cells that produce a monoclonal immunoglobulin in the bone marrow, causing end-organ damage such as hypercalcemia, renal failure, anemia, and osteolytic bone lesions [1]. It accounts for approximately $10 \%$ of all hematological malignancies, and the incidence is gradually increasing over time [2]. Survival has improved with the development of new and effective agents over the past two decades, but the disease remains incurable, and patients may experience various complications during treatment [3-5].

Infection is a major cause of morbidity and mortality during MM treatment. MM triggers a B-cell immune deficiency and hypogammaglobulinemia, increasing susceptibility to infection by encapsulated organisms [6]. Lymphopenia, neutropenia, or cytokines released by plasma cells worsen the immune defects [7-9]. Apart from the defects per se, advanced age, certain treatments, comorbidities, and catheter insertion can increase the infections in MM [10, 11]. In a population-based study with 9,253 patients with MM conducted in Sweden, those with MM were at 7-fold higher risk for infection than healthy participants, in which the risk was 11-fold greater during the first year after diagnosis [12]. Infection was a major cause of early mortality within 12 months in MM. In a previous study, $13.8 \%$ of patients died within 12 months of diagnosis; infection was the major cause $(36.2 \%)$ of death within 12 months after diagnosis [13]. Therefore, antibiotic prophylaxis is considered to decrease the infection rate and improve survival in MM. However, the effectiveness of antibiotic prophylaxis remains unclear in terms of the infection rate, emergence of resistant bacteria, and side effects. In addition, the most appropriate prophylactic antibiotics and their duration of use had received little attention.

Here, we evaluated the efficacy of levofloxacin prophylaxis in elderly patients with newly diagnosed MM who received bortezomib, melphalan, and prednisone (VMP) regimen. 


\section{MATERIALS AND METHODS}

\section{Patients}

We retrospectively analyzed the records of patients with newly diagnosed MM treated with VMP regimen between February 2011 and September 2020 at three institutes of the Republic of Korea. Patients with active infections at the time of diagnosis and those who commenced levofloxacin prophylaxis after the first cycle of VMP therapy were excluded. In addition, we excluded monoclonal gammopathy of undetermined significance, non-secretory MM, and plasma cell leukemia. The study was approved by the Institutional Review Boards of each participating institution and was conducted in accordance with the Declaration of Helsinki.

\section{Definitions}

The infectious complication grades were those of the National Cancer Institute Common Terminology Criteria (NCI-CTC) for Adverse Events (version 5.0). A grade 3 infection was a systemic infection requiring intravenous antibiotics, antifungal, or antiviral intervention. A grade 4 infection was lifethreatening. A grade 5 infection caused death. Grade 3-5 infection was classified as severe infection in this study. In addition, severe infection that occurred within the first four cycles was defined as early severe infection. The Charlson Comorbidity Index (CCI) was calculated at the time of diagnosis based on the clinical history, laboratory, and radiological tests. The age-adjusted CCI (ACCI) was calculated by adding the comorbidity score to the age score, which adds 1 point per decade to ages $>40$ years [14]. Treatment response was assessed on the first day of each cycle using the International Myeloma Working Group criteria [15]. Chromosomal abnormalities (CAs) were evaluated at the time of diagnosis using conventional cytogenetic studies or fluorescence in situ hybridization. A high-risk CA was defined when at least one of del(17p), t(4;14), or $\mathrm{t}(14 ; 16)$ was present.

\section{Statistical analysis}


Univariate analysis of factors associated with severe infection was performed using the $\chi^{2}$ test. Factors associated with $P$-values $<0.1$ were included in multivariate logistic regression analysis. Progression free survival (PFS) was calculated from the day of diagnosis to the day of disease progression or death from any cause. Overall survival (OS) was the period from the day of diagnosis to the date of the last follow-up or death from any cause. PFS and OS were evaluated using the Kaplan-Meier calculated and compared using the log-rank test. All statistical analyses were performed using the Statistical Package for the Social Sciences (SPSS) software (ver. 25; SPSS, Chicago, IL, USA). A $P$-value $<0.05$ was considered significant.

\section{RESULTS}

\section{Patient population}

A total of 258 patients were included. The clinical characteristics of the patients are summarized in

Table 1. The median age was 72 years (range, $64-86 \mathrm{yr}$ ), and $31.4 \%$ were aged $\geq 75$ years. Forty-one patients (15.9\%) were classified as International Staging System (ISS) I, 35.3\% as ISS II, and $48.1 \%$ as ISS III. Most patients (71.0\%) exhibited an Eastern Cooperative Oncology Group (ECOG) performance status (PS) of 0 or 1, 22.5\% an ECOG PS of 2, 4.7\% an ECOG PS of 3, and 1.9\% an ECOG PS of 4. A total of 132 patients (51.2\%) had comorbidities at the time of diagnosis. The median ACCI was 3 (range, 2-10). Of all patients, 57 (22.1\%) exhibited glomerular filtration rates $<30$ $\mathrm{mL} / \mathrm{min}$ at the time of diagnosis. Fourteen patients (5.4\%) were placed on hemodialysis at the time of diagnosis. Cytogenetic data were collected from 212 patients at the time of diagnosis, in which 38 (14.7\%) had evidenced of high-risk cytogenetics.

The median number of VMP cycles was 9 (range, 1-9), in which 133 patients (51.6\%) completed the 9 cycles. Of all patients, 204 (79.1\%) received levofloxacin prophylaxis during VMP therapy; however, $54(20.9 \%)$ were not prescribed with any antibiotic apart from an antiviral prophylaxis with oral acyclovir. In the levofloxacin prophylaxis group, most patients were prescribed once a day of $500 \mathrm{mg}$ 
tablet; however, $250 \mathrm{mg}$ tablet was prescribed for 21 patients because of decreased renal function. The median number of levofloxacin prophylaxis cycles was 4 (range, 1-9). The duration of levofloxacin prophylaxis was at the discretion of the clinician; however, 20 patients did not complete the planned levofloxacin prophylaxis. Six patients discontinued levofloxacin prophylaxis because of a generalized skin rash (5) or itching (1) during the first VMP cycle. Three patients discontinued levofloxacin prophylaxis because of gastrointestinal discomfort during the first or second VMP cycle. One patient stopped levofloxacin because of QT interval prolongation during the third VMP cycle. Ten patients stopped levofloxacin for unknown reasons. Trimethoprim-sulfamethoxazole (TMP-SMX) prophylaxis against Pneumocystis jirovecii pneumonia was prescribed for 50 patients in the levofloxacin group and for 47 patients who did not receive levofloxacin.

\section{Incidence and characteristics of severe infection}

Of all patients, 66 (25.5\%) experienced severe infections during VMP therapy, in which 5 developed two severe infections and 4 developed three severe infections. Therefore, the total was 79 severe infections over 1,709 cycles of VMP therapy. Overall, 69 patients (40.5\%) had grade 3, $4(5.1 \%)$ had grade 4 , and $6(7.6 \%)$ had grade 5 infections. The severe infection rates were somewhat higher in the no-prophylaxis than the levofloxacin prophylaxis group, but the difference was not significant $(33.3 \%$ vs. $23.5 \%, P=0.142$ ). Every episode of severe infections was summarized in Table 2. The most common was a respiratory infection [55 episodes (69.6\%)]. Twenty-three-episodes (29.2\%) yield pathogenic organisms in microbiological culture or polymerase chain reaction (PCR) testing, but most (70.8\%) did not. Most infections were treated with broad-spectrum antibiotics. Six patients died, including 4 in the no-levofloxacin group and 2 in the levofloxacin group. Two episodes of Clostridium difficile colitis developed in patients receiving levofloxacin. One episode of $P$. jiroveci pneumonia developed in a patient but not on TMP-SMX prophylaxis.

\section{Prognostic impact of early severe infection}


Fig. 1 shows the frequencies of severe infections by each cycle of VMP therapy in patients on levofloxacin prophylaxis or those who are not. Most infections $(74.7 \%)$ occurred in the first four cycles of therapy, regardless of levofloxacin prophylaxis status. The severe infection frequency was the highest during the first cycle and then decreased gradually. The early severe infection rate (within four cycles) was significantly lower in the levofloxacin prophylaxis group than the no-prophylaxis group $(17.6 \%$ vs. $31.5 \%, P=0.025)$.

We explored whether early severe infection affected survival. After overall median follow-up for 35.0 months (range, 0.4-110.1), the median PFS was 19.4 months [95\% Confidence Interval (CI), 17.121.8], whereas the median OS was not reached (Fig. 2A, B). Patients with early severe infections exhibited significantly inferior PFS and OS than those lacking such infections [PFS, 10.5 mo (95\% CI, 8.0-13.0) vs. 21.8 mo (95\% CI, 18.0-25.6), $P<0.001$; OS, 37.8 mo (95\% CI, 18.3-57.2) vs. not reached, $P<0.001$, Fig. 2C, D]. In univariate and multivariate analysis, early severe infection was an independent prognostic factor for PFS and OS (Supplementary Table 1).

\section{Factors associated with early severe infection}

Both univariate analysis and multivariate analyses revealed that three factors were significantly associated with early severe infection (Table 3): low serum levels of albumin $[\leq 3.5 \mathrm{~g} / \mathrm{dL}$; Odd Ratio (OR), 2.962; 95\% CI, 1.495-5.871; $P=0.002]$; deep response after VMP therapy ( $\geq$ a very good partial response; OR, 0.417 ; 95\% CI, 0.205-0.847; $P=0.016$ ); and levofloxacin prophylaxis (OR, $0.461 ; 95 \%$ CI, $0.220-0.964 ; P=0.040)$.

We also explored whether a recent model in predicting the risk of early severe infection [16] was effective in patients who were treated with VMP therapy. Of all patients, 134 (51.9\%) were classified as high-risk. Compared to the low-risk group, these patients did not differ significantly in terms of the early severe infection rate $(16.9 \%$ vs. $23.8 \%, P=0.168)$. However, in the subgroup analysis of patients not on levofloxacin prophylaxis, the high-risk group exhibited a significantly higher early severe 
infection rate than the low-risk group $(46.6 \%$ vs. $12.5 \%, P=0.007)$. In addition, in the subgroup analysis of the high-risk group, patients on levofloxacin prophylaxis exhibited a significantly lower early severe infection rate than patients not on levofloxacin ( $21.1 \%$ vs. $50 \%, P=0.002)$.

\section{DISCUSSION}

This real-world study found that severe infections in elderly patients with newly diagnosed MM were common during the first four cycles of VMP therapy, and such infections were significantly associated with poor survival. In general, elderly patients with MM may be more susceptible to infection than younger patients because of poor performance status, comorbidities, or sensitivity to toxicities. In addition, severe infection during treatment can trigger treatment delay or discontinuation; thus, affecting the outcomes. In the FIRST trial, severe infections were common in the first 4 months of treatment and significantly predicted poor OS [hazard ratio (HR), 9.1; 95\% CI, 5.6-14.6; $P<0.0001$ ] [16]. Therefore, prevention of early severe infection is important to allow maintenance of treatment and to improve survival outcomes in elderly with MM. In this study, most patients $(79.1 \%)$ received levofloxacin to prevent infection during VMP therapy, which decreased the early severe infection rate. The effectiveness of antibiotic prophylaxis in terms of preventing severe infections was not evident in a small, randomized, prospective trial, in which patients received daily ciprofloxacin or TMP-SMX during the first 2 months of treatment [17]. Most bacteria are now resistant to TMP-SMX [18, 19], in which ciprofloxacin does not counter Gram-negative infections well; thus, these agents may not prevent severe infection during $\mathrm{MM}$ treatment. In a recent large, multi-center, double-blinded, placebo-controlled, randomized, phase 3 trial, prophylactic levofloxacin during the first 12 weeks of anti-myeloma therapy significantly reduced febrile episodes and deaths compared to those in a placebo group [20]. However, the isolation rates of C. difficile, an extended-spectrum beta-lactamaseproducing Gram-negative bacteria, and methicillin-resistant Staphylococcus aureus did not differ between the two groups. Levofloxacin has potency against not only gram-negative bacteria but also 
gram-positive pathogens; thus, preventing severe infections during the first four therapy cycles in patients with MM.

In this study, seven viral infections (six caused by influenza) were confirmed by PCR testing, and some respiratory infections were clinically presumed to be viral. Influenza causes three to five million severe illnesses and approximately $290,000-650,000$ deaths annually, particularly in young children or elderly participants with comorbidities or who are immunosuppressed [21].

Several studies have reported that age, neutropenia, lymphopenia, disease burden, platelet count, and renal impairment predict infections in patients with $\mathrm{MM}[22,23]$. We found that a low serum level of albumin and a poor response to MM treatment were significantly associated with a higher severe infection rate. There is no confirmed predictor of infection in patients with MM; however, studies have differed in terms of primary endpoints, and the populations have been heterogeneous. Recently, a model in predicting the risk of early severe infection was developed in patients of the FIRST trial [16]. In that study, a scoring system based on serum levels of $\beta 2$-microglobulin, ECOG PS, hemoglobin, and lactate dehydrogenase levels indicated significantly different early severe infection rates $(24.0 \%$ vs. $7.0 \%, P<0.0001$ ) in those on (or not on) prophylaxis. In our study, this model predicted early severe infection in patients with MM, but not on levofloxacin. In addition, the early severe infection rate in the high-risk group predicted by the model was reduced by levofloxacin. Therefore, prophylactic levofloxacin should be prescribed in patients with MM.

Our work had certain limitations. Because of its retrospective nature, the numbers in the two groups differed. Patients who did not receive levofloxacin prophylaxis were relatively small. Physicians prescribed levofloxacin prophylaxis during induction therapy in the absence of any clear recommendation. The duration of levofloxacin was quite heterogeneous. Therefore, it was difficult to analyze the preventive effect of levofloxacin in this study. In addition, most pathogenic organisms were not identified; thus, we could not determine whether the incidence of severe bacterial infections was reduced by levofloxacin.

In conclusion, severe infections frequently developed during the first four cycles of VMP therapy in 
elderly patients with newly diagnosed MM. Early severe infection was significantly associated with poor survival. Levofloxacin significantly reduced the early severe infection rate. We suggest that levofloxacin prophylaxis is valuable during at least the first four cycles of VMP therapy in elderly patients with newly diagnosed MM.

\section{Authors' Disclosure of Potential Conflict of Interest}

No potential conflicts of interest relevant to this article were reported. 


\section{REFERENCES}

1. Kyle RA, Rajkumar SV. Multiple myeloma. Blood 2008;111:2962-72.

2. Siegel RL, Miller KD, Fuchs HE, Jemal A. Cancer statistics, 2021. CA Cancer J Clin 2021;71:7-33.

3. Kristinsson SY, Landgren O, Dickman PW, Derolf AR, Björkholm M. Patterns of survival in multiple myeloma: a population-based study of patients diagnosed in Sweden from 1973 to 2003. J Clin Oncol 2007;25:1993-9.

4. Kumar SK, Rajkumar SV, Dispenzieri A, et al. Improved survival in multiple myeloma and the impact of novel therapies. Blood 2008;111:2516-20.

5. Kumar SK, Dispenzieri A, Lacy MQ, et al. Continued improvement in survival in multiple myeloma: changes in early mortality and outcomes in older patients. Leukemia 2014;28:11228.

6. Backhaus E, Berg S, Andersson R, et al. Epidemiology of invasive pneumococcal infections: manifestations, incidence and case fatality rate correlated to age, gender and risk factors. BMC Infect Dis 2016;16:367.

7. Mills KH, Cawley JC. Abnormal monoclonal antibody-defined helper/suppressor T-cell subpopulations in multiple myeloma: relationship to treatment and clinical stage. $\mathrm{Br} \mathrm{J}$ Haematol 1983;53:271-5.

8. Schütt $\mathrm{P}$, Brandhorst D, Stellberg $\mathrm{W}$, et al. Immune parameters in multiple myeloma patients: influence of treatment and correlation with opportunistic infections. Leuk Lymphoma 2006;47:1570-82.

9. Frassanito MA, Cusmai A, Dammacco F. Deregulated cytokine network and defective Th1 immune response in multiple myeloma. Clin Exp Immunol 2001;125:190-7.

10. Gavazzi G, Krause KH. Ageing and infection. Lancet Infect Dis 2002;2:659-66.

11. Nucci M, Anaissie E. Infections in patients with multiple myeloma in the era of high-dose therapy and novel agents. Clin Infect Dis 2009;49:1211-25. 
12. Blimark C, Holmberg E, Mellqvist UH, et al. Multiple myeloma and infections: a populationbased study on 9253 multiple myeloma patients. Haematologica 2015;100:107-13.

13. Jung SH, Cho MS, Kim HK, et al. Risk factors associated with early mortality in patients with multiple myeloma who were treated upfront with a novel agents containing regimen. BMC Cancer 2016;16:613.

14. Charlson M, Szatrowski TP, Peterson J, Gold J. Validation of a combined comorbidity index. J Clin Epidemiol 1994;47:1245-51.

15. Kumar S, Paiva B, Anderson KC, et al. International Myeloma Working Group consensus criteria for response and minimal residual disease assessment in multiple myeloma. Lancet Oncol 2016;7:e328-e46.

16. Dumontet C, Hulin C, Dimopoulos MA, et al. A predictive model for risk of early grade $\geq 3$ infection in patients with multiple myeloma not eligible for transplant: analysis of the FIRST trial. Leukemia 2018;32:1404-13.

17. Vesole DH, Oken MM, Heckler C, et al. Oral antibiotic prophylaxis of early infection in multiple myeloma: a URCC/ECOG randomized phase III study. Leukemia 2012;26:2517-20.

18. Smilack JD. Trimethoprim-sulfamethoxazole. Mayo Clin Proc 1999;74:730-4.

19. Zhanel GG, Karlowsky JA, Harding GK, et al. A Canadian national surveillance study of urinary tract isolates from outpatients: comparison of the activities of trimethoprimsulfamethoxazole, ampicillin, mecillinam, nitrofurantoin, and ciprofloxacin. The Canadian Urinary Isolate Study Group. Antimicrob Agents Chemother 2000;44:1089-92.

20. Drayson MT, Bowcock S, Planche T, et al. Levofloxacin prophylaxis in patients with newly diagnosed myeloma (TEAMM): a multicentre, double-blind, placebo-controlled, randomised, phase 3 trial. Lancet Oncol 2019;20:1760-72.

21. Iuliano AD, Roguski KM, Chang HH, et al. Estimates of global seasonal influenza-associated respiratory mortality: a modelling study. Lancet 2018;391:1285-300.

22. Jung SH, Bae SY, Ahn JS, et al. Lymphocytopenia is associated with an increased risk of 
severe infections in patients with multiple myeloma treated with bortezomib-based regimens. Int J Hematol 2013;97:382-7.

23. Soekojo CY, Low JZ, Oh J, Ooi M, De Mel S, Chng WJ. Bacterial infection among patients with multiple myeloma treated with bortezomib-based induction therapy: real-world experience in an Asian Cancer Center. Clin Lymphoma Myeloma Leuk 2020;20:e165-e70. 
Table 1. Baseline clinical characteristics of all patients $(\mathrm{N}=258)$.

\begin{tabular}{|c|c|}
\hline \multicolumn{2}{|l|}{ Variables } \\
\hline Median age, years (range) & $72(64-86)$ \\
\hline$\geq 75$-year, N (\%) & $81(31.4)$ \\
\hline Male, N (\%) & $135(52.3)$ \\
\hline ECOG PS $\geq 2, N(\%)$ & $75(29.1)$ \\
\hline \multicolumn{2}{|c|}{ Immunoglobulin (Ig) type, N (\%) } \\
\hline $\operatorname{IgG}$ & $153(59.3)$ \\
\hline $\operatorname{Ig} \mathrm{A}$ & $65(25.2)$ \\
\hline $\operatorname{IgM}$ & $3(1.2)$ \\
\hline Light chain only & $34(13.2)$ \\
\hline Missing & $3(1.2)$ \\
\hline \multicolumn{2}{|c|}{ International Staging System, N (\%) } \\
\hline I & $41(15.9)$ \\
\hline II & $91(35.3)$ \\
\hline III & $124(48.1)$ \\
\hline Missing & $2(0.8)$ \\
\hline \multicolumn{2}{|l|}{ ACCI, N (\%) } \\
\hline 2 & $44(17.1)$ \\
\hline 3 & $93(36.0)$ \\
\hline 4 & $67(26.0)$ \\
\hline 5 or more & $54(20.9)$ \\
\hline Immunoparesis, N (\%) & $193(74.8)$ \\
\hline $\mathrm{LDH}>(1 \times \mathrm{ULN}), \mathrm{N}(\%)$ & $53(20.5)$ \\
\hline $\mathrm{GFR}<30 \mathrm{~mL} / \mathrm{min} / 1.73 \mathrm{~m}^{2}$ & $57(22.1)$ \\
\hline ANC $<1,000 \times 10^{9} / \mathrm{L}, \mathrm{N}(\%)$ & 11 (4.3.) \\
\hline $\mathrm{ALC}<800 \times 10^{9} / \mathrm{L}, \mathrm{N}(\%)$ & $27(10.5)$ \\
\hline \multicolumn{2}{|l|}{ Cytogenetics, N (\%) } \\
\hline High & $38(14.7)$ \\
\hline Standard & $174(67.4)$ \\
\hline Not evaluable & $46(17.8)$ \\
\hline
\end{tabular}

${ }^{\dagger}$ : Immunoparesis is defined as suppression in the levels of 1 or 2 uninvolved immunoglobulin.

Abbreviations: ACCI, Age-adjusted Charlson Comorbidity Index; ALC, absolute lymphocyte count; ANC, absolute neutrophil count; ECOG, Eastern Cooperative Oncology Group; GFR, glomerular filtration rate; LDH, lactate dehydrogenase; N, number; PS, performance status; ULN, upper limit of normal value. 
Table 2. Summary of severe infections developed during bortezomib, melphalan, and prednisone therapy.

\begin{tabular}{|c|c|}
\hline \multicolumn{2}{|l|}{ Type of infection, N (\%) } \\
\hline Respiratory & $55(69.6)$ \\
\hline Urinary tract & $5(6.3)$ \\
\hline Gastrointestinal & $10(12.6)$ \\
\hline Hepatobiliary & $4(5.1)$ \\
\hline Sepsis/bacteremia & $1(1.3)$ \\
\hline Skin/soft tissue & $2(2.5)$ \\
\hline Joint & $1(1.3)$ \\
\hline Unknown & $1(1.3)$ \\
\hline \multicolumn{2}{|l|}{ Pathogenic microorganism, N (\%) } \\
\hline Streptococcus pneumoniae & $2(2.5)$ \\
\hline Klebsiella pneumonia & $2(2.5)$ \\
\hline Escherichia coli & $3(3.8)$ \\
\hline Staphylococcus aureus, methicillin-susceptible & $1(1.3)$ \\
\hline Nocardia farcinica & $1(1.3)$ \\
\hline Bacillus species & $1(1.3)$ \\
\hline Burkholderia & $1(1.3)$ \\
\hline Enterococcus faecalis & $1(1.3)$ \\
\hline Influenza type $A$ & $5(6.3)$ \\
\hline Influenza type B & $1(1.3)$ \\
\hline Respiratory syncytial virus & $1(1.3)$ \\
\hline Candida krusei & $1(1.3)$ \\
\hline Pneumocystitis jiroveci & $1(1.3)$ \\
\hline Clostridium difficil ${ }^{a)}$ & $2(2.5)$ \\
\hline Unknown & $56(70.8)$ \\
\hline
\end{tabular}

${ }^{a}$ Two cases in the levofloxacin prophylaxis group. 
Table 3. Univariate and multivariate analyses of risk factors in early severe infection during bortezomib, melphalan, and prednisone therapy.

\begin{tabular}{|c|c|c|c|c|c|}
\hline \multirow[t]{2}{*}{ Variable } & \multicolumn{3}{|c|}{ Univariate analysis } & \multicolumn{2}{|c|}{ Multivariate analysis } \\
\hline & $\begin{array}{c}\text { Infection rate, } \\
\mathrm{N}(\%)\end{array}$ & $P$ & OR $(95 \% \mathrm{CI})$ & $P$ & OR $(95 \% \mathrm{CI})$ \\
\hline \multicolumn{6}{|l|}{ Age } \\
\hline$\geq 75$ years & $21(25.9)$ & 0.148 & $1.586(0.847-2.970)$ & & \\
\hline \multicolumn{6}{|l|}{ Gender } \\
\hline Female & $24(19.5)$ & 0.696 & $0.886(0.483-1.625)$ & & \\
\hline \multicolumn{6}{|l|}{ ECOG PS } \\
\hline$\geq 2$ & $21(28.0)$ & 0.058 & $1.835(0.975-3.453)$ & 0.469 & $1.298(0.64-2.626)$ \\
\hline \multicolumn{6}{|l|}{ Immunoparesis } \\
\hline Yes & $41(21.2)$ & 0.938 & $1.030(0.487-2.176)$ & & \\
\hline \multicolumn{6}{|l|}{ ACCI } \\
\hline$\geq 4$ & $28(23.1)$ & 0.332 & $1.349(0.736-2.471)$ & & \\
\hline \multicolumn{6}{|l|}{ ISS } \\
\hline III & $25(20.2)$ & 0.954 & $0.982(0.534-1.806)$ & & \\
\hline \multicolumn{6}{|l|}{$\mathrm{CrCl}$} \\
\hline$<30 \mathrm{~mL} / \mathrm{min}$ & $12(21.1)$ & 0.914 & $1.041(0.505-2.145)$ & & \\
\hline \multicolumn{6}{|l|}{$\mathrm{LDH}$} \\
\hline High & $15(28.3)$ & 0.093 & $1.809(0.900-3.636)$ & 0.100 & $1.868(0.888-3.929)$ \\
\hline \multicolumn{6}{|l|}{$\mathrm{S} \beta 2 \mathrm{MG}$} \\
\hline$\geq 5.5 \mathrm{mg} / \mathrm{L}$ & $27(21.4)$ & 0.731 & $1.112(0.608-2.035)$ & & \\
\hline \multicolumn{6}{|l|}{ Serum albumin } \\
\hline$<3.5 \mathrm{~g} / \mathrm{dL}$ & $38(29.9)$ & $<0.001$ & $3.302(1.709-6.378)$ & 0.002 & $2.962(1.495-5.871)$ \\
\hline \multicolumn{6}{|l|}{ ANC } \\
\hline$<1,000 / \mu \mathrm{L}$ & $3(27.3)$ & 0.572 & $1.478(0.378-5.772)$ & & \\
\hline \multicolumn{6}{|l|}{ ALC } \\
\hline$<800 / \mu \mathrm{L}$ & $5(18.5)$ & 0.783 & $0.866(0.312-2.407)$ & & \\
\hline \multicolumn{6}{|l|}{ Hemoglobin } \\
\hline$\leq 11 \mathrm{~g} / \mathrm{dL}$ & $46(22.0)$ & 0.228 & $1.693(0.713-4.019)$ & & \\
\hline \multicolumn{6}{|l|}{ Spinal fracture } \\
\hline Yes & $28(23.3)$ & 0.360 & $1.327(0.724-2.434)$ & & \\
\hline \multicolumn{6}{|l|}{ Best response } \\
\hline$\geq$ VGPR & $14(12.3)$ & 0.003 & $0.377(0.193-0.736)$ & 0.016 & $0.417(0.205-0.847)$ \\
\hline \multicolumn{6}{|c|}{ TMP-SMX use } \\
\hline Yes & $24(24.7)$ & 0.195 & $1.496(0.812-2.759)$ & & \\
\hline \multicolumn{6}{|l|}{ Levofloxacin } \\
\hline Yes & $36(17.6)$ & 0.025 & $0.466(0.237-0.919)$ & 0.040 & $0.461(0.220-0.964)$ \\
\hline
\end{tabular}

Abbreviations: ACCI, Age-adjusted Charlson Comorbidity Index; ALC, absolute lymphocyte count; ANC, absolute neutrophil count; $\mathrm{CrCl}$, creatinine clearance; ECOG, Eastern Cooperative Oncology Group; ISS, International Staging System; LDH, lactate dehydrogenase; PS, performance status; S $\beta 2 \mathrm{MG}$, serum $\beta 2-$ microglobulin; TMP-SMX, trimethoprim-sulfamethoxazole; VGPR, very good partial response. 
Figure legends

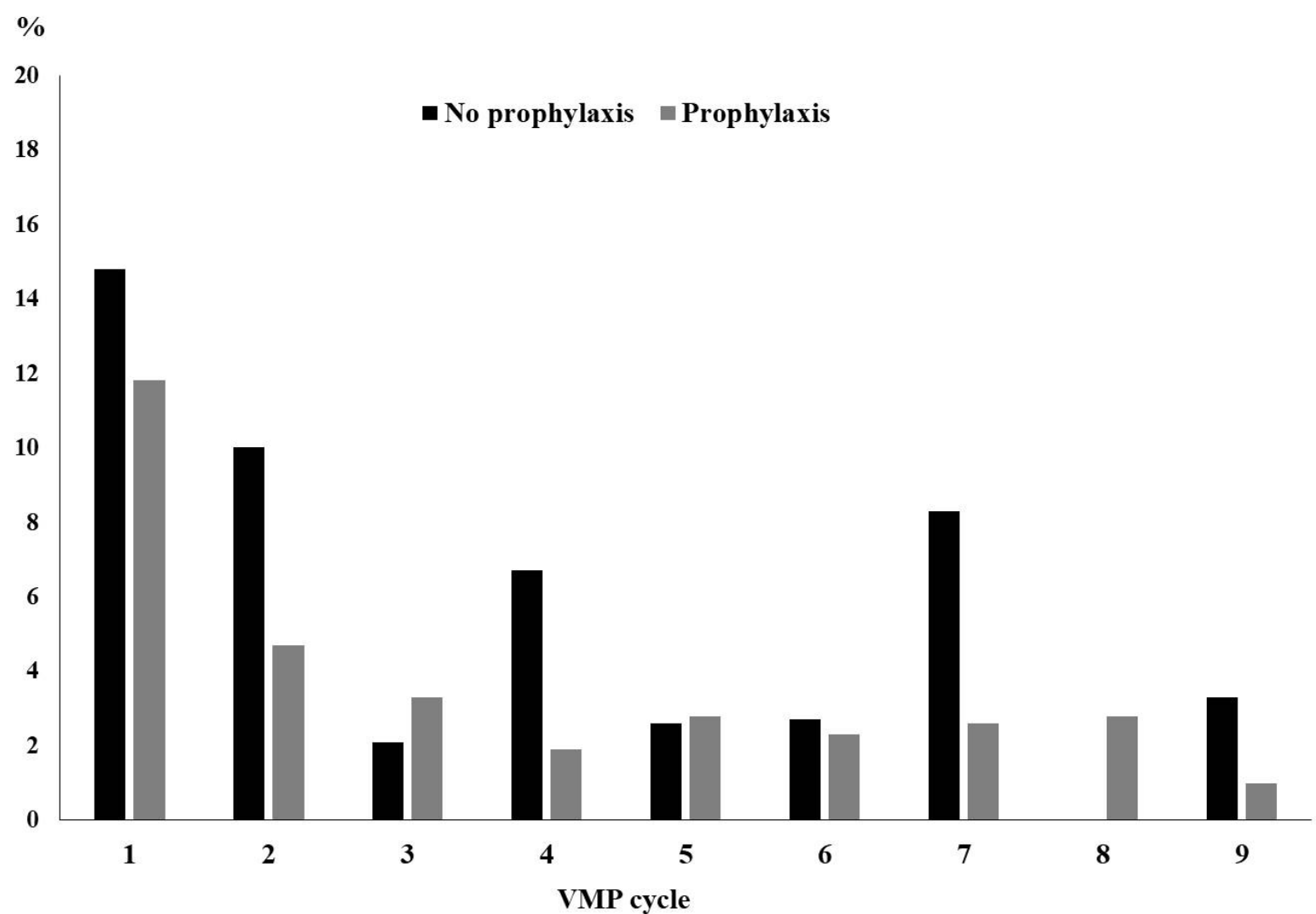

Fig. 1. Rates of severe infection by levofloxacin prophylaxis status of patients during bortezomib, melphalan, and prednisone (VMP) therapy. 

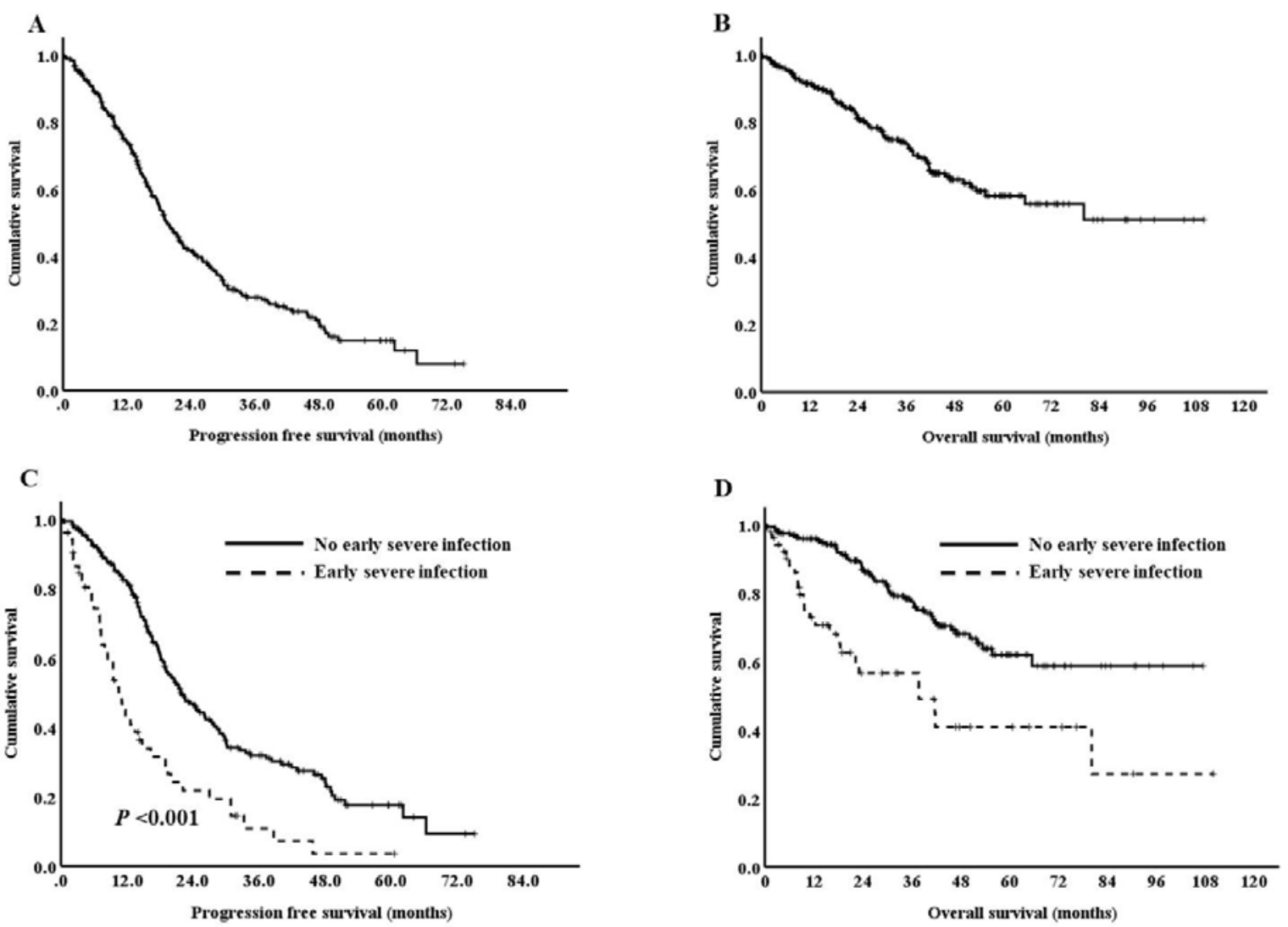

Fig. 2. Kaplan-Meier curves for progression-free survival (PFS) and overall survival (OS) in all patients (A, B) and those who experienced early severe infections $(\mathbf{C}, \mathbf{D})$. 
Supplementary table 1. Factors influencing progression free survival (PFS) and overall survival (OS) in all patients.

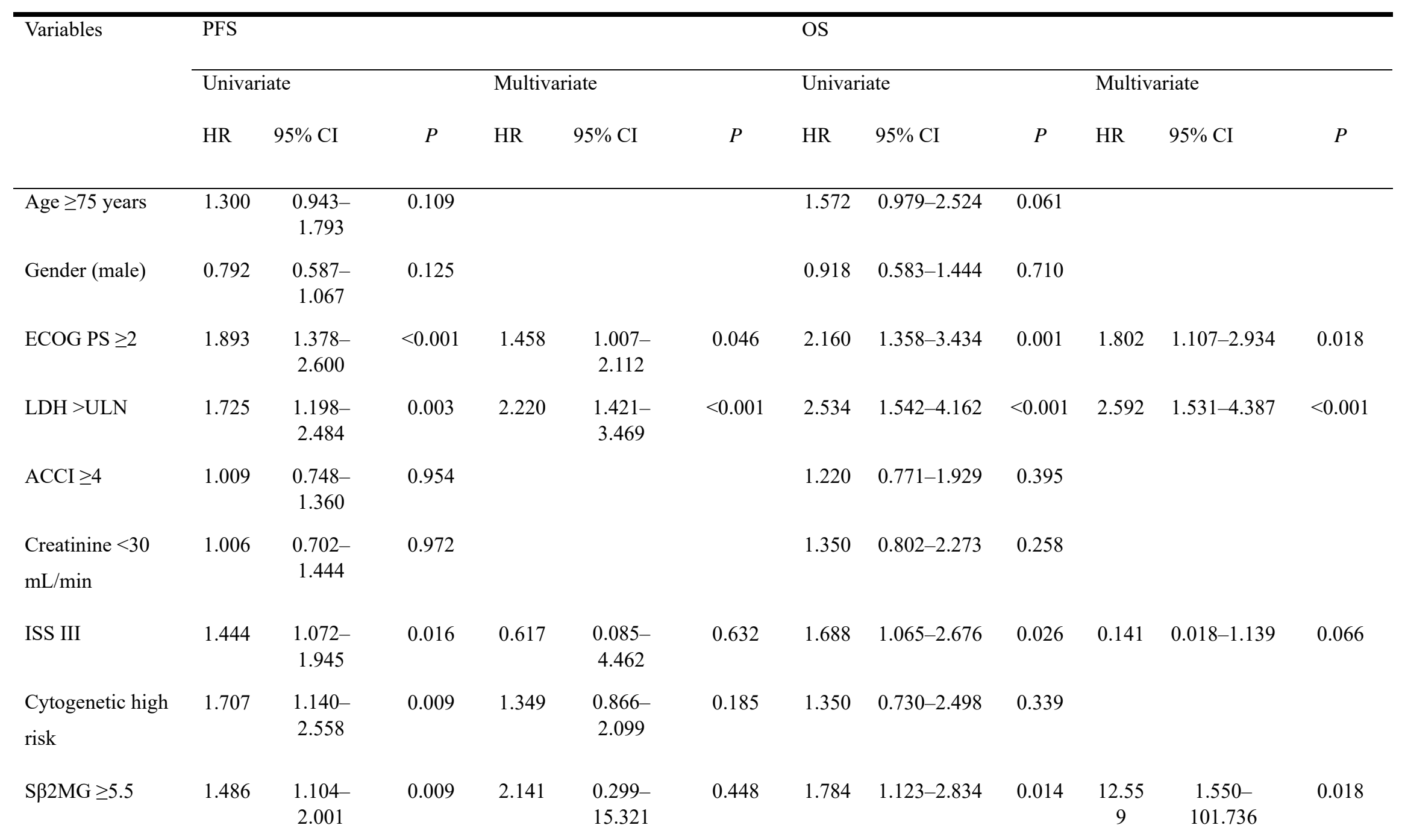


$\mathrm{mg} / \mathrm{L}$

\begin{tabular}{|c|c|c|c|c|c|c|c|c|c|c|c|c|}
\hline $\begin{array}{l}\text { Albumin }<3.5 \\
\mathrm{~g} / \mathrm{dL}\end{array}$ & 1.985 & $\begin{array}{c}1.464- \\
2.691\end{array}$ & $<0.001$ & 1.890 & $\begin{array}{l}1.302- \\
2.743\end{array}$ & 0.001 & 2.025 & $1.264-3.247$ & 0.003 & 1.759 & $1.062-2.913$ & 0.028 \\
\hline $\begin{array}{l}\text { Early severe } \\
\text { infection }\end{array}$ & 2.349 & $\begin{array}{l}1.659- \\
3.327\end{array}$ & $<0.001$ & 1.768 & $\begin{array}{l}1.160- \\
2.695\end{array}$ & 0.008 & 2.672 & $1.641-4.352$ & $<0.001$ & 2.083 & $1.220-3.556$ & 0.007 \\
\hline $\begin{array}{l}\text { Beast response } \\
\geq \mathrm{VGPR}\end{array}$ & 0.421 & $\begin{array}{c}0.310- \\
0.572\end{array}$ & $<0.001$ & 0.415 & $\begin{array}{c}0.291- \\
0.592\end{array}$ & $<0.001$ & 0.330 & $0.198-0.552$ & $<0.001$ & 0.329 & $0.193-0.563$ & $<0.001$ \\
\hline
\end{tabular}

Abbreviations: ACCI, Age-adjusted Charlson Comorbidity Index; CI, confidence interval; ECOG PS, Eastern Cooperative Oncology Group performance status; HR, hazard ratio; ISS, International Staging System; LDH, lactate dehydrogenase; S $\beta 2 M G$, serum $\beta 2$-microglobulin; ULN, upper limit of normal; VGPR, very good partial response. 